\title{
Gastro-resistant Capsule, Hard Dosage Form
}

National Cancer Institute

\section{Source}

National Cancer Institute. Gastro-resistant Capsule, Hard Dosage Form. NCI Thesaurus. Code C149531.

Solid single-dose, delayed-release preparation contained in a hard shell. The preparation is intended to resist the gastric fluid and to release the active substance(s) in the intestinal fluid. Hard gastro-resistant capsules are usually made by filling hard capsules with gastro-resistant granules or solid particles made gastro-resistant by coating or, in certain cases, by providing hard capsules with a gastro-resistant shell. They are intended for oral use. 